\title{
Probiotics improve atopic dermatitis in children and adolescents: a double blind, placebo-controlled study.
}

\author{
Paula Danielle Santa Maria Albuquerque de Andrade ${ }^{1}$, Jorgete Maria e Silva ${ }^{1}$, Vanessa \\ Carregaro $^{1}$, Laís Sacramento ${ }^{1}$, Luciana Roberti ${ }^{1}$, Davi Aragon ${ }^{1}$, and Pérsio Roxo-Junior ${ }^{1}$ \\ ${ }^{1}$ Universidade de São Paulo Faculdade de Medicina de Ribeirão Preto
}

May 12, 2020

\begin{abstract}
Background: The hypothesis that early stimulation of the gut microbiota contributes to immune system balance has encouraged the use of probiotics to treat atopic dermatitis (AD), an immunological disorder characterized by chronic and relapsing skin inflammation, in several clinical studies. This study aimed to evaluate the clinical efficacy of a mixture of probiotics (Lactobacillus and Bifidobacterium) in children and adolescents with $\mathrm{AD}$ and the effects of probiotics on sensitization, inflammation, and immunological tolerance. Methods: In this double-blind, randomized, placebo-controlled clinical trial, children and adolescents (aged 2 to 19 years) received one gram (sachet) per day of probiotics or placebo for six months. SCOring of AD (SCORAD) index, serum IgE levels, skin prick test, tolerogenic and inflammatory cytokines were evaluated. Results: Forty patients were evaluated and clinical response was significantly better in the probiotic group as compared to the placebo group after treatment for six months; SCORAD decreased (95\% CI, 2.44-52.94) even after adjustment for co-variables (95\% CI, 5.52-59.13). Three months after the treatment was discontinued, improvement persisted in the probiotic group even after adjustment for co-variables (95\% CI, 0.78- 27.70). IgE levels, skin prick test and cytokines did not differ between groups. Conclusions: AD children and adolescents treated with a mixture of probiotics (Lactobacillus rhamnosus, Lactobacillus acidophilus, Lactobacillus paracasei, and Bifidobacterium lactis) for six months presented a significate SCORAD reduction as compared to placebo group. This probiotics mixture did not affect SPT and IgE levels, as well as inflammatory or tolerogenic cytokines. ClinicalTrials.gov \#NCT02519556.
\end{abstract}

\section{Introduction}

Atopic dermatitis $(\mathrm{AD})$ is a chronic predominantly type 2 inflammatory skin disease characterized by intense pruritus and associated with atopic comorbidities ${ }^{1}$.

AD diagnosis is based on clinical symptoms and Scoring for Atopic Dermatitis index (SCORAD) helps physicians to assess severity on a regular basis ${ }^{2}$.

Several studies have demonstrated a close relationship between microbial dysbiosis and development of allergy during childhood ${ }^{3}$. The composition of the gut microbiome, including the presence of Bifidobacterium andLactobacillus species, is important for health-promoting effects ${ }^{4}$ and probiotics, live microorganisms whose adequate administration benefits the host's health ${ }^{5}$, have been attractive in the treatment of allergic diseases ${ }^{6}$.

This study aimed to evaluate the clinical benefit of combined probiotics in the management of children and adolescents with $\mathrm{AD}$. In addition, other assessed parameters were sensitization (serum IgE levels and skin prick test), inflammation (IFN-g, IL-1 $\beta$, IL-4, IL-6, IL-8, IL-17, TNF- $\alpha$ ), and tolerance (IL-10 and TGF- $\beta$ ).

\section{Methods}

Study design (Figure 1) 
This was a double-blind, randomized and placebo-controlled clinical trial involving children and adolescents aged between 6 months to 19 years with mild, moderate or severe AD, diagnosed according to the Hanifin\&Hajka criteria ${ }^{7}$. Consecutive patients were invited to participate and then they were clinically assessed for AD severity (on the basis of SCORAD) and submitted to laboratorial analysis and to skin prick test (SPT). Patients were stratified in mild, moderate, or high severity groups. Blocks of four (04) patients were considered in each severity group for random allocation, according to a list of computer-generated random numbers. Each group had the same amount of patients receiving placebo or probiotics. Patients were enrolled until August 2015, when they started the treatment, and they were followed up for one year. The recruitment, randomization, clinical assessments, laboratory analysis and SPT were performed by the same investigator.

\section{Patient population}

Patients followed up at the Pediatric Allergy Outpatient Clinics in a tertiary hospital and that presented at least one documented flare were included in the study. Patients were excluded if they had other skin diseases, which could interfere; oral corticosteroid or immunosuppressant use within 30 days; lose over $50 \%$ of the assessments conducted along the trial.

\section{Sample size}

The determination of the sample size required for the study was based on the SCORAD mean comparison at the end of the follow-up, between probiotics and placebo groups, considering a parallel group trial. Establishing a variance of 10.2 points and a clinically relevance difference of 8.7 points, as well as the significance level of 0.05 and statistical power of 0.90 , required a total of 60 patients to conduct the study.

\section{Intervention}

A formula containing a mixture of 4 probiotics strains (Probiatop@):Lactobacillus rhamnosus HN001 - $10^{9}$ Colony Forming Units (CFU); Lactobacillus acidophilus NCFM - $10^{9}$ CFU; Lactobacillus paracasei Lcp-37 - $10^{9} \mathrm{CFU}$; and Bifidobacterium lactis HN019 - $10^{9} \mathrm{CFU}$, was used in the dose of one gram (one sachet) per day. Placebo consisted of one gram of maltodextrin, in the powder form, which was administered as a sachet to ensure similarity to the probiotic. Probiotic and placebo sachets were identical, except for their identification as group A or B, to guarantee that participants and investigator were blind to their contents. Patients were instructed to dilute one sachet in $100 \mathrm{~mL}$ of natural water and to take it every morning on a daily basis. Treatment lasted the first six months of the trial. A committee composed of three physicians who were not involved in the trial ensured external monitoring.

\section{Assessments}

Clinical assessments were conducted every three months and blood samples for laboratory analysis were collected before the trial start, after the treatment and at the end of the follow up. The skin prick tests were carried out at the end of the follow up.

For cytokines analysis, peripheral blood was collected and serum was obtained by centrifugation at 2500 $\mathrm{rpm}$ and $4^{\circ} \mathrm{C}$ for $10 \mathrm{~min}$. The serum samples were stored and frozen at $-80 \operatorname{deg} \mathrm{C}$ until all analyses were performed. In the end, the wells of a 96-well plate were covered and incubated with purified antibodies anti-IL-17 (R\&D System, Minneapolis, MN, EUA), anti-IFN- $\gamma$, anti-IL-1 $\beta$, anti-IL-4, anti-IL-6, anti-IL-8, anti-IL-10, anti-TNF- $\alpha$, or anti-TGF- $\beta$ (BD Bioscience, San Diego, CA, EUA) and enzyme-linked immune sorbent assay (ELISA) was performed according to the manufacturer's instructions.

Serum total IgE levels were measured by a fluoroenzyme immunoassay (Phadia ImmunoCap System, Uppsala, Sweden), according to the manufacturer's instructions. The results were expressed in $\mathrm{kU} / \mathrm{L}$ and were considered elevated when they were higher than $100 \mathrm{kU} / \mathrm{L}$.

Skin prick test was performed in all patients using a panel of standardized extracts (Greer@): mites (Dermatophagoides pteronyssinus, Dermatophagoides farinae and Blomia tropicalis ); cockroachs (Blatella germanica andPeriplaneta americana); pet's dander (cat and dog) and some food allergens (milk, egg, soybean, 
wheat, peanuts, seafood and fish). The test was considered positive when the diameter of the wheal was greater than $3 \mathrm{~mm}$ emerged $20 \mathrm{~min}$ after application.

\section{Ethics considerations}

The study (ClinicalTrials.gov \#NCT02519556) was approved by the Ethics Committee of Hospital das Clínicas de Ribeirão Preto (University of São Paulo). Parents provided a written informed consent for their child's participation; adolescents signed the assent term.

\section{Statistical analysis}

The SCORAD was represented as percentage. The values observed at times 3, 6, 9, and 12 months were divided by the value observed at T0 and then multiplied by 100. Data analysis was made by adjusting simple and multiple linear mixed models, that considers the dependence between measurements for the same individual along the trial.

Statistical analysis of the variable serum IgE was similar than performed for SCORAD.

To verify the association of the intervention groups with the cytokines and the SPT, log-binomial regression models were proposed and raw and adjusted relative risks and their $95 \%$ confidence intervals for the categories improvement and worsening of symptoms.

All statistical analysis was performed with software SAS 9.4.

\section{Results}

\section{Baseline description of patients}

Sixty patients were recruited and randomized in two groups (Placebo and Probiotics) and twenty patients were discontinued (Figure 2). Table 1 shows the characteristics of both groups after recruitment phase.

Characteristics of patients at baseline were demonstrated in the Table 2. Mild SCORAD was between 5.5 and 23.9 (mean 14.7), moderate SCORAD was between 25.4 and 49.8 (mean 37.6) and severe SCORAD was between 50.3 and 85.5 (mean 67.9).

\section{Clinical assessment}

After six months of treatment (T6), probiotic group presented a significant reduction in SCORAD as compared with control group ( $\mathrm{p}=0.03$; CI 95\%, 2.44-52.94), as observed in Figure 3. This beneficial effect persisted after the adjustment for the co-variables age, sex, delivery type, and medications (moisturizers, anti-histamines, topical corticosteroids, topical immunosuppressants, and antileukotrienes) ( $\mathrm{p}=0.02$; CI $95 \%, 5.52-59.13)$.

The improvement in SCORAD persisted three months more after treatment has been discontinued (T9), even after adjustment for the co-variables ( $\mathrm{p}=0.04$; CI 95\%, 0.78-27.70), when compared to the probiotic group itself. However, the clinical assessment between T9 and T12 showed a significant increase in SCORAD ( $\mathrm{p}=0.04$; CI 95\%, -28.71-0.68) in probiotic group.

\section{Sensitization assessment}

Regarding serum IgE levels, the groups did not differ significantly (Table 3). Regarding to SPT, relative risk was not significantly different between the groups.

\section{Tolerance and inflammation assessment}

Regarding to tolerogenic and inflammatory cytokines, relative risk was not significantly different between the groups.

\section{Discussion}


This randomized, double-blind and placebo-controlled trial evaluated the clinical and laboratorial efficacy of a mixture of probiotics containing Lactobacillus rhamnosus, Lactobacillus acidophilus ,Lactobacillus paracasei , and Bifidobacterium lactis in children and adolescents with AD. After treatment for six months, SCORAD decreased in the probiotic group as compared to the placebo group $(\mathrm{p}=0.03)$, even after adjustment for co-variables $(\mathrm{p}=0.02)$. However, the groups did not differ regarding to inflammatory or tolerogenic cytokines, SPT, or serum IgE levels. To our knowledge, this is the first Brazilian randomized, double-blind and placebo-controlled trial that has shown clinical benefits of probiotics in patients with AD.

Allergic diseases prevalence has increased in recent times, in both high and low-resource countries ${ }^{8,9}$. Because a high morbidity of $\mathrm{AD}^{10}$, it is necessary to find an accessible, safe, and effective treatment, which can minimize and/or override the skin inflammation long-term effects. There is growing evidence that the relation between microbiota and immune response can influence in this disease ${ }^{11}$. In general, combined administration of probiotics (before and after birth) has been beneficial, and Lactobacillus rhamnosus $G G$ andBifidobacterium seen to be the most efficient strains ${ }^{12}$.

Han et al. ${ }^{13}$ performed a randomized, double-blind, placebo-controlled study lasted twelve weeks to evaluate the influence of Lactobacillus plantarum CJLP133 in children with AD aged between 1 and 12 years. The authors showed SCORAD improvement and a significant decrease in IFN- $\gamma$, IL-4 and eosinophil's number. In other study, Wang et al. ${ }^{14}$ evaluated the effect of Lactobacillus paracasei alone, Lactobacillus fermentumalone, and the association of both strains in AD patients. Four months after the treatment was discontinued, the authors observed reduction of SCORAD in all groups that received probiotics as compared to the placebo group. Huang et al. ${ }^{15}$ carried out a recent meta-analysis including thirteen randomized clinical assays (n $=1070$ patients) and demonstrated that probiotics may have the potential to decrease SCORAD values in children with AD. Recently, Navarro-Lopez et. al. ${ }^{16}$ published a double-blind, placebo-controlled clinical trial conducted with children and adolescents between 4 and 17 years of age with moderate AD. Patients were supplemented daily with a mixture of probiotics composed by Bifidobacterium lactis CECT 8145, Bifidobacterium longum CECT 7347, and Lactobacillus casei CECT 9104 for 12 weeks. The authors showed reduction in SCORAD and use of topical corticosteroids.

Our study is in accordance with meta-analysis of Huang et al. ${ }^{15}$ and with the other aforementioned doubleblind, placebo-controlled studies. We found a significant reduction of SCORAD after treatment with a mixture of probiotics (Lactobacillus andBifidobacterium) for six months as compared to the placebo group. The reduction of SCORAD persisted until three months after treatment was discontinued, suggesting a short-term beneficial effect. Probably this clinical beneficial effect did not persist until the end of the study because treatment was stopped shortly. Other possibility is patient's microbiota has been already developed by various host- and environment-related factors. Some studies demonstrate that around three years old, the microbiota composition resembles the microbiota composition in adults ${ }^{17}$.

Two main biological pathways are involved in pathogenesis of AD: epidermal epithelial dysfunction and modifications in innate/adaptive immune response ${ }^{11}$. T helper cells are particularly important in inflammation; they determine the immune response degree and direction. T regulatory cells (Treg) are essential to limit excessive immune-inflammatory response. Reduced Treg number in early life is a risk factor for later AD development ${ }^{10}$. In the acute phase of $\mathrm{AD}$, Th2 cell-mediated immune response triggers the inflammatory process. On the other hand, Th1 cell-mediated immune response predominates in the chronic phase of the disease. Some evidences have suggested that Th17 cells are also involved in AD pathogenesis ${ }^{18}$. Probiotic bacteria can affect dendritic cells and modify the T helper cells balance ${ }^{10}$. Previous studies have shown that probiotics inhibit dendritic cell maturation and hence decrease naive T cells differentiation into Th2 cells ${ }^{19,20}$. Kim et al. ${ }^{21,22}$ demonstrated that probiotics reduce allergic disease expression in mice by acting in dendritic cells. Holowacz et al. ${ }^{23}$ showed that a mixture of probiotics reduced significantly chronic inflammation in rat skin, as judged from the lower levels of pro-inflammatory cytokines (IL-1 $\beta$, IL-6, TNF- $\alpha$, IL-17, and IL-22) and higher levels of tolerogenic cytokines, such as IL-10.

However, results concerning the effect of probiotics on $\mathrm{T}$ cells subgroups and cytokines in children with $\mathrm{AD}$ are conflicting. Some studies have reported a tendency toward the Th1 profile resulting in increased inter- 
feron gamma (IFN $\gamma)$ production, whereas other studies have not detected any effect on Th1/Th2 balance or Treg ${ }^{10}$. Yeşilova et al. ${ }^{24}$ performed a double-blind, randomized, placebo-controlled clinical assay to investigate how eight-week treatment with a combination of probiotics (Bifidobacterium bifidum, Lactobacillus acidophilus, Lactobacillus casei and Lactobacillus salivarius ) affected children with AD aged between 1 and 13 years old. They concluded that the probiotics effectively reduced SCORAD and serum levels of IL-5, IL-6, IFN- $\gamma$ and IgE, while IL-2, IL-4, TNF- $\alpha$, and IL-10 remained unaltered. On the other hand, Ludwing et al. ${ }^{25}$ verified thatLactobacillus rhamnosus GG soluble mediators (LSM) did not modify the number or the maturation stage of dendritic cells in healthy donors; nevertheless, these cultivated cells induced IFN- $\gamma$ and IL-2 production in TCD4+ and CD25+ cells.

In our study, probiotics and placebo groups did not differ in acting on inflammatory (IFN- $\gamma$, IL-1 $\beta$, IL- 4 , IL-6, IL-8, IL-17, and TNF- $\alpha$ ) or tolerogenic (IL-10 and TGF- $\beta$ ) cytokines. Although we did not carry out cell culture, our evaluation included a panel of cytokines that represent all possible immune responses (Th1, Th2, Th17, and Treg), which led us to infer that probiotics did not interfere the cell subtypes. We can point possible reasons for these results. Yeşilova et al. ${ }^{24}$ used a higher dose of probiotics than we did $(2 \mathrm{~g}$ $\mathrm{x} 1 \mathrm{~g}$ ). Besides that, they analyzed cytokines in the plasma whereas we analyzed cytokines in the serum. Nevertheless, studies analyzing cytokines in humans with AD and treated with probiotics are scarce, which prevents us from drawing more conclusions about other possible factors influencing our results.

Similarly to cytokines, probiotics and placebo groups did not differ in acting on serum IgE levels or SPT results. We did not find studies that evaluated SPT in AD patients treated with probiotics.

Probiotics, especially lactobacilli and bifidobacteria ${ }^{26}$, are known to be safe $^{27}$. The adverse effects most commonly described are diarrhea, vomiting, and increased flatulence ${ }^{27}$. However, invasive infections have been observed in immunocompromised adults, but are rare in breastfed infants ${ }^{26}$. We did not verify any adverse effect in the group of patients that used probiotics.

Strengths of this study are that no clinical trials explored the effect of Bifidobacteria in combination with Lactobacillus in children. Moreover, the safety profile of probiotic treatment was not adequately studied, accordingly to a recent systematic review and meta-analysis performed by Zhao et al. ${ }^{28}$

The main limitations of the present study include the small sample size and the difficult of patients' adhesion. These difficulties could be justified by the participants' age range. Parents usually feel insecure and question the need to subject their children to the study. Furthermore, children's acceptance may be more difficult, mainly among younger patients. This can justify why the number of clinical assays involving children is smaller than other age ranges.

More studies on the immune response and the intestinal microbiota during use of probiotics will help to understand $\mathrm{AD}$ pathogenesis and to elucidate the controversial results obtained in different studies, thereby contributing to alternative therapies.

\section{Conclusion}

$\mathrm{AD}$ children and adolescents treated with a probiotics mixture (Lactobacillus rhamnosus HN001 - $10^{9}$ CFU;Lactobacillus acidophilus NCFM - $10^{9}$ CFU;Lactobacillus paracasei Lcp-37 - $10^{9}$ CFU; andBifidobacterium lactis HN019 - $10^{9} \mathrm{CFU}$ ) for six months presented a significate SCORAD reduction as compared to placebo group. SCORAD reduction persisted for three months after treatment has been discontinued. This probiotics mixture did not affect SPT and IgE levels, as well as inflammatory (IFN-g, IL-1 $\beta$, IL-4, IL-6, IL-8, IL-17, and TNF- $\alpha$ ) or tolerogenic cytokines (IL-10 and TGF- $\beta$ ).

\section{Acknowledgments}

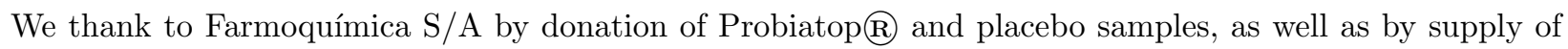
cytokine kits.

\section{Impact statement}


A recent meta-analysis showed that only Lactobacillus strains were protective in the treatment of AD in children. Only few studies investigated the effect of Bifidobacterium species. No clinical trials explored the effect of Bifidobacteria in combination withLactobacillus . Moreover, safety profile of probiotic treatment was not adequately studied. Our study evaluated the effect ofLactobacillus in combination with Bifidobacteria in $\mathrm{AD}$ children and we addressed the occurrence of side effects.

\section{References}

1. Simpson EL, Paller AS, Siegfried EC, et al. Efficacy and Safety of Dupilumab in Adolescents With Uncontrolled Moderate to Severe Atopic Dermatitis: A Phase 3 Randomized Clinical Trial. JAMA Dermatol. 2019.

2. Antunes AA, Solé D, Carvalho VO, et al. Updated practical guide on atopic dermatitis - Part I: etiopathogenesis, clinical features, and diagnosis. Joint position paper of the Brazilian Association of Allergy and Immunology and the Brazilian Society of Pediatrics. Arq Asma Alerg Imunol. 2017;1(2):131-56.

3. Dzidic M, Abrahamsson TR, Artacho A, et al. Oral microbiota maturation during the first 7 years of life in relation to allergy development. Allergy. 2018;73(10):2000-11.

4. Cai Y, Folkerts J, Folkerts G, Maurer M, Braber S. Microbiota-dependent and -independent effects of dietary fibre on human health. Br J Pharmacol. 2020;177(6):1363-81.

5. Fiocchi A, Pawankar R, Cuello-Garcia C, et al. World Allergy Organization-McMaster University Guidelines for Allergic Disease Prevention (GLAD-P): Probiotics. World Allergy Organ J. 2015;8(1):4.

6. Azad MAK, Sarker M, Wan D. Immunomodulatory Effects of Probiotics on Cytokine Profiles. Biomed Res Int. 2018;2018:8063647.

7. Hanifin JM, Rajka G. Diagnostic features of atopic dermatitis. Acta Dermato vener. (Stockh). 1980;92:44-47.

8. Plummer EL, Chebar LA, Tobin JM, et al. Postnatal probiotics and allergic disease in very preterm infants: Sub-study to ProPrems randomized trial. Allergy. 2020;75(1):127-136.

9. Avershina E, Cabrera Rubio R, Lundgard K, et al. Effect of probiotics in prevention of atopic dermatitis is dependent on the intrinsic microbiota at early infancy. J Allergy Clin Immunol. 2017;139(4):1399-402 e8.

10. Ro ADB, Simpson MR, Ro TB, et al. Reduced Th22 cell proportion and prevention of atopic dermatitis in infants following maternal probiotic supplementation. Clin Exp Allergy. 2017;47(8):1014-21.

11. Huang YJ, Marsland BJ, Bunyavanich S, et al. The microbiome in allergic disease: Current understanding and future opportunities-2017 PRACTALL document of the American Academy of Allergy, Asthma \& Immunology and the European Academy of Allergy and Clinical Immunology. J Allergy Clin Immunol. 2017;139(4):1099-110.

12. Blattner CM, Crosby MS, Goedken M, et al. Update: Do probiotics prevent or treat pediatric atopic dermatitis? Pediatr Allergy Immunol. 2016;27(4):425-8.

13. Han Y, Kim B, Ban J, et al. A randomized trial of Lactobacillus plantarum CJLP133 for the treatment of atopic dermatitis. Pediatr Allergy Immunol. 2012;23(7):667-73.

14. Wang IJ, Wang JY. Children with atopic dermatitis show clinical improvement after Lactobacillus exposure. Clin Exp Allergy. 2015;45(4):779-87.

15. Huang R, Ning H, Shen M, Li J, Zhang J, Chen X. Probiotics for the Treatment of Atopic Dermatitis in Children: A Systematic Review and Meta-Analysis of Randomized Controlled Trials. Front Cell Infect Microbiol. 2017;7:392.

16. Navarro-Lopez V, Ramirez-Bosca A, Ramon-Vidal D, et al. Effect of Oral Administration of a Mixture of Probiotic Strains on SCORAD Index and Use of Topical Steroids in Young Patients With Moderate Atopic Dermatitis: A Randomized Clinical Trial. JAMA Dermatol. 2018;154(1):37-43.

17. Pinto MC. Intestinal microbiota homeostasis: health or disease in human [monography]. Coimbra: Pharmacy School of University of Coimbra; 2016.

18. Saeki H, Kabashima K, Tokura Y, et al. Efficacy and safety of ustekinumab in Japanese patients with severe atopic dermatitis: a randomized, double-blind, placebo-controlled, phase II study. Br J Dermatol. 2017;177(2):419-27. 
19. Kwon HK, Lee CG, So JS, et al. Generation of regulatory dendritic cells and CD4+Foxp3+ T cells by probiotics administration suppresses immune disorders. Proc Natl Acad Sci U S A. 2010;107(5):2159-64.

20. Weiss G, Christensen HR, Zeuthen LH, et al. Lactobacilli and bifidobacteria induce differential interferon-beta profiles in dendritic cells. Cytokine. 2011;56(2):520-30.

21. Kim HJ, Kim HY, Lee SY, et al. Clinical efficacy and mechanism of probiotics in allergic diseases. Korean J Pediatr. 2013;56(9):369-76.

22. Kim HJ, Kim YJ, Lee SH, et al. Effects of Lactobacillus rhamnosus on asthma with an adoptive transfer of dendritic cells in mice. J Appl Microbiol. 2013;115(3):872-9.

23. Holowacz S, Guinobert I, Guilbot A, et al. A Mixture of Five Bacterial Strains Attenuates Skin Inflammation in Mice. Antiinflamm Antiallergy Agents Med Chem. 2018;17(2):125-37.

24. Yesilova Y, Calka O, Akdeniz N, et al. Effect of probiotics on the treatment of children with atopic dermatitis. Ann Dermatol. 2012;24(2):189-93.

25. Ludwig IS, Broere F, Manurung S, et al. Lactobacillus rhamnosus GG-Derived Soluble Mediators Modulate Adaptive Immune Cells. Front Immunol. 2018;9:1546.

26. Vandenplas Y, Huys G, Daube G. Probiotics: an update. J Pediatr (Rio J). 2015;91(1):6-21.

27. Awasthi S, Wilken R, Patel F, et al. Dietary supplementation with Bifidobacterium longum subsp. infantis (B. infantis) in healthy breastfed infants: study protocol for a randomised controlled trial. Trials. 2016;17(1):340.

28. Zhao M, Shen C, Ma L. Treatment efficacy of probiotics on atopic dermatitis, zooming in on infants: a systematic review and meta-analysis. Int J Dermatol. 2018;57(6):635-41.

\section{Tables}

Table 1 - Characteristics of patients after recruitment.

\begin{tabular}{lll}
\hline & Placebo group & Probiotic group \\
\hline Participants & 30 & 30 \\
$\begin{array}{l}\text { Sex Female } \\
\text { Male }\end{array}$ & 1614 & 1713 \\
$\begin{array}{l}\text { Age range at the start of the study Breastfed infants } \\
2-6 \text { years }\end{array}$ & 210126 & 09138 \\
$6-12$ years & & \\
$\begin{array}{l}\text { Adolescents } \\
\text { Delivery type Normal }\end{array}$ & 921 & \\
$\begin{array}{l}\text { Cesarean section } \\
\text { Initial SCORAD Mild } \\
\text { Moderate }\end{array}$ & 13125 & 14124 \\
Severe & & \\
\hline
\end{tabular}

Table 2 - Characteristics of patients at baseline.

\begin{tabular}{lll}
\hline & Placebo group & Probiotic group \\
\hline Participants & 16 & 24 \\
Sex Female & 88 & 168 \\
Male & & 07107 \\
Age range at the start of the study Breastfed infants 2-6 years & 0493 & \\
$6-12$ years & & 113 \\
Adolescents & 511 & \\
$\begin{array}{l}\text { Delivery type Normal } \\
\text { Cesarean section }\end{array}$ & &
\end{tabular}




\begin{tabular}{lll}
\hline & Placebo group & Probiotic group \\
\hline $\begin{array}{l}\text { Initial SCORAD Mild } \\
\text { Moderate }\end{array}$ & 763 & 1293 \\
Severe & & \\
\hline
\end{tabular}

Table 3 - Serum IgE: comparison between Placebo (A) and Probiotic (B) groups.

\begin{tabular}{llllllll}
\hline & \multicolumn{3}{c}{ Simple Model } & Simple Model & & Adjusted Model & Adjusted Mo \\
\hline & & $95 \%$ CI & $95 \%$ CI & & & $95 \%$ CI & $95 \%$ CI \\
Comparisons & Mean difference & $\mathrm{p}$ & LL & UL & Mean difference & p & LL \\
A6 - B6 & 0,04 & 0.93 & -0.82 & 0.90 & $-0,03$ & 0.95 & -0.88 \\
A12 - B12 & 0,48 & 0.27 & -0.38 & 1.33 & 0,41 & 0.33 & -0.43 \\
\hline
\end{tabular}

$\mathrm{LL}=$ Lower limit. $\mathrm{UL}=$ Upper limit.

\begin{tabular}{|c|c|c|c|}
\hline$>\quad 2015$ & $\begin{array}{l}\text { After a } \\
\text { baseline } \\
\text { (TO) }\end{array}$ & & up \\
\hline - Enrollment & $\begin{array}{l}\text { - Clinical } \\
\text { assessment } \\
\text { - Laboratory } \\
\text { analysis } \\
\text { - Skin prick tests }\end{array}$ & $\begin{array}{l}\text { - Placebo } \\
\text { - Probiotics }\end{array}$ & $\begin{array}{l}\text { - T3: Clinical } \\
\text { assessment } \\
\text { - T6: Clinical } \\
\text { assessment and } \\
\text { laboratory analysis } \\
\text { - T9: Clinical } \\
\text { assessment } \\
\text { - T12: Clinical } \\
\text { assessment, } \\
\text { laboratory analysis } \\
\text { and skin prick tests }\end{array}$ \\
\hline
\end{tabular}



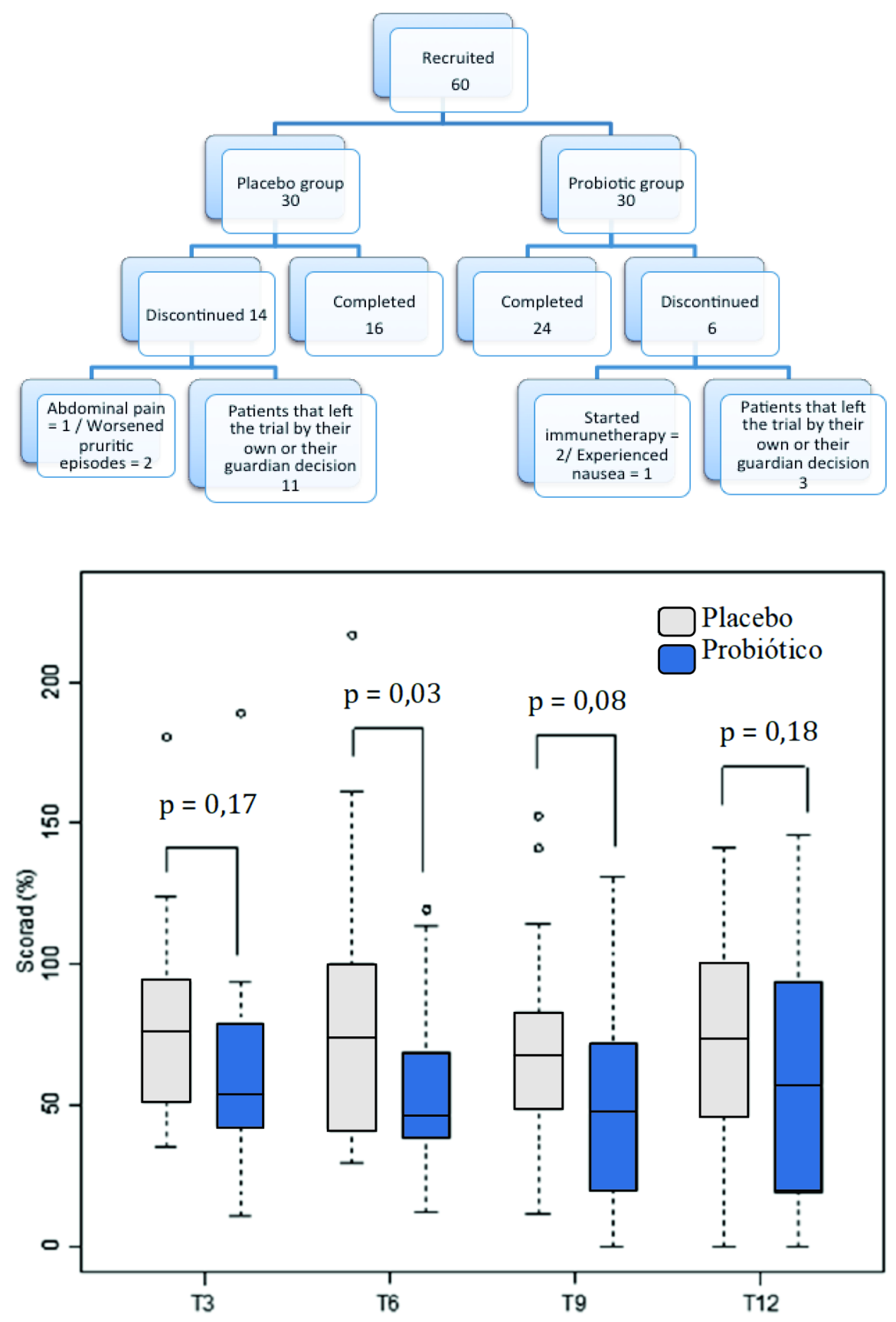